\title{
Narrativa
}

\section{Monete false. Le contraddizioni del capitalismo occidentale nella narrativa di Elvira Dones e Anilda Ibrahimi}

\section{Claudia Carmina}

\section{(2) OpenEdition Journals \\ Edizione digitale \\ URL: https://journals.openedition.org/narrativa/330 \\ DOI: $10.4000 /$ narrativa.330 \\ ISSN: 2804-1224 \\ Editore \\ Presses universitaires de Paris Nanterre}

\section{Edizione cartacea}

Data di pubblicazione: 1 décembre 2020

Paginazione: 141-152

ISBN: 978-2-84016-381-7

ISSN: $1166-3243$

Notizia bibliografica digitale

Claudia Carmina, «Monete false. Le contraddizioni del capitalismo occidentale nella narrativa di Elvira Dones e Anilda Ibrahimi», Narrativa [Online], 42 | 2020, online dal 01 décembre 2021, consultato il 08 décembre 2021. URL: http://journals.openedition.org/narrativa/330 ; DOI: https://doi.org/10.4000/ narrativa.330 


\section{Monete false. \\ Le contraddizioni del capitalismo occidentale nella narrativa di Elvira Dones e Anilda Ibrahimi}

\section{RIASSUNTO}

Il saggio analizza il modello economico occidentale in quanto oggetto di narrazione in Vergine giurata di Elvira Dones e Rosso come una sposa di Anilda Ibrahimi. Questi due romanzi, pubblicati rispettivamente nel 2007 e nel 2008, reinterpretano da un punto di vista "diverso" le questioni economiche, dando voce a una alterità che non è solo geografica, e appuntandosi sulle contraddizioni del capitalismo globale. In queste narrazioni il mito della ricchezza occidentale, che attraverso la retorica dei media colonizza l'immaginario migrante, si traduce in smascheramento.

\section{RÉSUMÉ}

Cet article analyse le modèle économique occidental en tant qu'objet de narration dans Vergine giurata de Elvira Dones et Rosso come una sposa de Anilda Ibrahimi. Ces deux romans, publiés respectivement en 2007 et 2008, réinterprètent, selon un point de vue "autre", les questions économiques, en donnant la parole à une diversité qui n'est pas que géographique et en mettant en évidence les contradictions du capitalisme global. Dans ces narrations, le mythe de la richesse occidentale, qui colonise l'imaginaire migrant par le biais de la rhétorique des médias, se traduit en dévoilement.

$$
\begin{gathered}
*^{*} * \\
\text { 'YOUNG LADIES DON'T UNDERSTAND POLITICAL ECONOMY". } \\
\text { DONNE, ECONOMIA, STEREOTIPI }
\end{gathered}
$$

"Young ladies don't understand political economy", sentenzia Mr Brooke nelle prime pagine di Middlemarch di George Eliot, liquidando così, senza discuterle, le opinioni della nipote Dorothea in materia di investimenti e di sviluppo.

1. Eцioт George, Middlemarch [1874], London, Wordsworth Classics, 1994, p. 14. 
Le donne non s'intendono di economia: questo pregiudizio di genere è avvalorato indirettamente da un altro persistente luogo comune secondo cui l'economia è una scienza sans entrailles ${ }^{2}$, per usare la nota formula flaubertiana, e pertanto è tradizionalmente declinata al maschile. Del resto il modello occidentale dell'homo oeconomicus si lega a un'identità di genere precisa e ineludibile: è maschio come Robinson Crusoe, ci avverte Gillian J. Hewitson ${ }^{3}$. Il Robinson di Defoe, com'è noto, costituisce l'incarnazione esemplare del "rational economic man", cioè di uno dei più persistenti "miti dell'individualismo moderno"4. È il personaggio-alfiere di una visione egemonica del mondo caratterizzata dall'asservimento della natura, dal primato della logica economica e dal trionfo dell'individualismo.

Nel romanzo Foe di Joseph Michael Coetzee, pubblicato nel 1986, la vicenda di Robinson viene destrutturata e rielaborata problematicamente, in una rinegoziazione dei significati complessa e ambivalente che gioca sull'“interstizialità" sulla sovrapposizione e sull'interferenza. La protagonista è una donna, Susan Barton, che, a seguito di un naufragio, approda su un'isola sperduta abitata solo dal signor Cruso e dal suo fedele servitore Friday. Lo smascheramento delle contraddizioni del capitalismo imperialista passa qui attraverso il rovesciamento della prospettiva narrativa che coincide con l'assunzione di un nuovo protagonismo femminile. Nell'isola su cui approda Susan un'altra economia è possibile, un'economia in cui il lavoro non sia più finalizzato alla produzione e al profitto: a differenza del Robinson di Defoe, Cruso non è interessato a imporre il suo

2. Flaubert Gustave, Dizionario dei luoghi comuni [1910], tr. it. di J. Rodolfo Wilcock, Milano, Adelphi, 1980, p. 27. Su questa lapidaria definizione di Flaubert cfr. LOMBARDI Chiara, "Dalla parte dell'uomo. L'economia nella letteratura e nelle scienze umane", in CoSMo. Comparative Studies in Modernim, n. 4, 2014, p. 5.

3. Cfr. Hewitson Gillian J., Feminist Economics: interrogating the masculinity of rational economic man, Cheltenham, Edward Elgar, 1999; GraPARD Ulla, Hewitson Gillian J., Robinson Crusoe's economic man: a construction and deconstruction, London and New York, Routledge, 2011.

4. Cfr. Watt Ian, Miti dell'individualismo moderno. Faust, don Chisciotte, don Giovanni, Robinson Crusoe [1996], Roma, Donzelli Editore, 2007, pp. 123-240. Per un'analisi del personaggio di Robinson Crusoe come antesignano di una "tendenza dinamica del capitalismo stesso il cui scopo non è mai il mantenimento dello status quo" (ID., Le origini del romanzo borghese. Studi su Defoe, Richardson e Fielding [1957], Milano, Bompiani, 1980, p. 61), si rileggano, oltre agli scritti dello stesso Watt, anche: MORETTi Franco, Il borghese. Tra storia e letteratura [2013], Torino, Einaudi, 2017, e Rizzoli Renato, Letteratura ed economia. Intrecci discorsivi nella cultura inglese della modernità e della postmodernità, Alessandria, Edizioni dell'Orso, 2014.

5. Riprendo la nozione di "interstizialità" da Homi K. Bhabha. Cfr. BнAвHA Homi K., I luoghi della cultura [1994], tr. it. di Antonio Perri, Roma, Meltemi Editore, 2001. 
dominio sull'isola, si nutre quasi esclusivamente di lattuga e di uova di uccello, e addirittura sembra aver smarrito il senso del tempo, tant'è che non sente l'esigenza di tenere un diario. E proprio per sopperire a questa mancanza Susan vuole raccontarne la storia. Eppure l'esperienza di Susan non è pubblicamente raccontabile al femminile. La sua voce è condannata a restare subalterna, come subalterno e condannato al mutismo è il personaggio del colonizzato, Friday. Se il silenzio di Friday è una forma opaca ma ostinata di resistenza che non si lascia penetrare né interpretare, la storia di Susan viene invece manipolata dallo scrittore Mr. Foe: perché la narrazione acquisti lo statuto di "libro", e cioè possa trasformarsi in un prodotto commercializzabile sul mercato editoriale, le tracce della presenza e dell'autorialità femminile vanno cancellate. Alla fine Foe è la storia di un romanzo che non può essere scritto.

La voce della donna, il silenzio irriducibile e incomprensibile di Friday, la violenza epistemica di Mr. Foe che falsifica il racconto per trasformarlo in merce: nell'ambiguità del gioco metaletterario il romanzo di Coetzee si struttura al modo di una contronarrazione postcoloniale ${ }^{6}$ che pone al lettore una domanda cruciale. È la stessa domanda che fa da titolo al fortunato saggio di Gayatri Spivak: Can the Subaltern Speak ? ? I subalterni, e soprattutto le subalterne, possono parlare? "La subalterna come femmina non può essere udita o letta. [...] La subalterna non può parlare"": nel 1988 Spivak conclude il suo saggio con questa presa di posizione asseverativa, per poi riaprire la questione, riformulandone la risposta dieci anni più tardi. "Anche noi siamo nativi. Parliamo come il Venerdì di Defoe, solo molto meglio", scrive nel 1999 Spivak a chiusura della rilettura del Foe di Coetzee condotta in Critica della ragione postcoloniale, e continua: "Dopotutto sono passati quasi trecento anni dalla creazione di Venerdì da parte di Defoe. L'imperialismo territoriale, allora appena agli inizi, ha ceduto il posto al neocapitalismo e adesso alla globalizzazione9 ${ }^{9}$ Oggi, a distanza di più di

6. Per la definizione di Foe come romanzo "postcoloniale" cfr. Dolce Maria Renata, "Decostruzione e dialogo con il canone: J. M. Coetzee riscrive Robinson Crusoe in Foe", in Quaderni. Dipartimento di Lingue e Letterature Straniere, vol. 21/1999, Lecce, 2004, pp. 1-50.

7. Spivak Gayatri Chakravorty, "Can the Subaltern Speak?", in GrossberG Lawrence, Nelson Cary, Treichler Paula A. (a cura di), Marxism and The Interpretation of Culture, London, Macmillan, 1988, pp. 271-313. Sulla questione cfr. Di Maio Alessandra, "Subaltern Studies", Studi culturali, http://www.studiculturali.it/ dizionario/lemmi/subaltern_studies.html.

8. SpIvak Gayatri Chakravorty, "Can the Subaltern Speak?”, cit., p. 63. La traduzione è mia.

9. EAD., Critica della ragione postcoloniale. Verso una storia del presente in dissolvenza [1999], a cura di Patrizia Cefalato, Roma, Meltemi Editore, 2004. 
vent'anni dalla pubblicazione di Critica della ragione postcoloniale, va constatato che chi vive una condizione di doppia subalternità, come donna e straniera, non solo rivendica il diritto di parlare ma forza le forme tradizionali per esprimere il senso contrappuntistico di una differenza, mettendo in dubbio le gerarchie e raccontando un mondo in transizione.

\section{LO SGUARDo DELL'Altra: L'IMMAGINARIO ECONOMICO DELL'OCCIDENTE NEI ROMANZI DELLE SCRITTRICI DELLA DIASPORA ALBANESE}

Le scritture di donne occupano un posto di rilievo all'interno della "letteratura mondo italiana", per utilizzare l'appropriata formula proposta da Rosanna Morace in esplicito omaggio a Édouard Glissant ${ }^{10}$. In questa nebulosa testuale dai confini sfrangiati i testi delle scrittrici in lingua italiana della diaspora albanese, come Anilda Ibrahimi, Elvira Dones e Ornela Vorpsi, costituiscono un raggruppamento coeso e riconoscibile. Mettendo a fuoco un'identità autoriale instabile che si costruisce sempre in rapporto allo sguardo dell'altro - il lettore -, queste narrazioni, scritte da donne migranti di prima generazione e popolate di personaggi femminili, interpretano da un punto di vista 'diverso' le grandi questioni economiche, dando voce a un'alterità che non è solo geografica, ma anche esistenziale e biologica.

Nell'analizzare la produzione di queste scrittrici ho scelto di concentrarmi sui romanzi d'esordio in lingua italiana ${ }^{11}$ che appaiono in concomitanza o

10. Cfr. Morace Rosanna, Letteratura-mondo italiana, Pisa, ETS, 2012. La questione terminologica, che riguarda la difficoltà di definire la letteratura italiana della migrazione con una formula unica ma necessariamente problematica, si lega a una complessa questione ermeneutica, discussa già a partire dagli anni Novanta in Italia, dove il dibattito critico sul tema è stato aperto dagli studi di Gnisci (GNISCI Armando, Il rovescio del gioco, Roma, Carocci, 1992; ID., La letteratura italiana della migrazione, Roma, Lilith, 1998). Per apprezzare l'abbondanza delle scritture di donne tra le "opere degli autori translingue italiani” (MORACE Rosanna, Letteratura-mondo italiana, cit., p. 9), è possibile consultare i dati riportati in BASILI\&LIMM (http://basili-limm.el-ghibli. it), la Banca dati degli Scrittori Immigrati in Lingua Italiana e della Letteratura Italiana della Migrazione Mondiali, fondata da Armando Gnisci nel 1997 e rinnovata nel 2017. Ricciarda Ricorda ha messo in evidenza la particolare "fecondità delle scritture femminili in questo ambito", sottolineando come "accanto al dato statistico, merita di essere segnalata anche la crescente maturità critica delle scrittrici, il valore di eccellenza raggiunto da alcune di esse". Cfr. RicORDA Ricciarda, "Scrittrici della migrazione in Italia", in SERAfin Silvana (a cura di), Donne al caleidoscopio. La riscrittura dell'identità femminile nei testi dell'emigrazione, Oltreoceano, vol. 7, 2013, p. 28.

11. Anilda Ibrahimi è residente a Roma dal 1997 e ha scelto l'italiano come lingua letteraria per tutti i suoi romanzi. La sua prima opera è appunto il romanzo Rosso come 
negli immediati dintorni della crisi finanziaria: Vergine giurata di Elvira Dones e Rosso come una sposa di Anilda Ibrahimi, pubblicati rispettivamente da Feltrinelli e da Einaudi nel 2007 e nel 2008. Sono appunto gli anni della crisi finanziaria: i primi segnali di cedimento del mercato immobiliare iniziano a manifestarsi nel 2005; ma è nel 2007 che la crisi esplode negli Stati Uniti e si diffonde per contagio e con estrema velocità fino a coinvolgere le principali economie mondiali. La recessione mette in luce le debolezze e i rischi del capitalismo liberista e produce una riflessione sulle trasformazioni storiche dell'homo oeconomicus governato ora, come teorizza Gallino, dalla "megamacchina" del "finanzcapitalismo":

Il finanzcapitalismo è una mega-macchina che è stata sviluppata nel corso degli ultimi decenni allo scopo di massimizzare e accumulare, sotto forma di capitale e insieme di potere, il valore estraibile sia dal maggior numero possibile di esseri umani, sia dagli ecosistemi. L'estrazione di valore tende ad abbracciare ogni momento e aspetto dell'esistenza degli uni e degli altri, dalla nascita alla morte o all'estinzione. Come macchina sociale, il finanzcapitalismo ha superato ciascuna delle precedenti, compresa quella del capitalismo industriale, a motivo della sua estensione planetaria e della sua capillare penetrazione in tutti i sottosistemi sociali, e in tutti gli strati della società, della natura e della persona ${ }^{12}$.

Le narrazioni di Dones e Ibrahimi non affrontano di petto il tema della crisi finanziaria dei subprime, ma permettono di inquadrarlo nel più ampio contesto di una "onnimercificazione"13 del mondo, guardando da un'ottica eccentrica

una sposa. Elvira Dones, invece, ha attraversato l'Italia e l'italiano per approdare in nuove terre: Dones lascia l'Albania nel 1988 e si traferisce prima nel Canton Ticino (dove impara l'italiano) e poi negli USA, per tornare in Svizzera nel 2015. Vergine giurata è il primo romanzo che scrive in italiano, dopo aver composto i libri precedenti in albanese. Nella produzione in albanese Dones affronta questioni e temi, quali la violenza, il trauma della migrazione, l'identità femminile, il rapporto tra culture ed economie diverse, che poi ritornano, rielaborati, in Vergine giurata. Sono stati tradotti dall'albanese in italiano i romanzi Senza bagagli [1997] (tr. it. di Alba Molla, Nardò, Besa Editrice, 1998), Sole bruciato [2000] (tr. it. di Elio Miracco, Milano, Feltrinelli, 2001), Bianco giorno offeso [2001], tradotto dalla stessa Elvira Dones (Novara, Interlinea, 2004) e I mari ovunque del 2004 (tr. it. di Rovena Troqe, Novara, Interlinea, 2007). Nel 2011 è poi apparso il romanzo Piccola guerra inutile (Torino, Einaudi, 2011), scritto in italiano come Vergine giurata.

12. Gallino Luciano, Finanzcapitalismo. La civiltà del denaro in crisi, Torino, Einaudi, 2011, p. 5.

13. A impiegare il concetto di "onnimercificazione" è Karl Polanyi, che nei suoi studi ha analizzato il passaggio dall'economia mercantile "sostanziale" a un'economia estensiva, dominata dalla "grande finanziarizzazione", in cui il capitale non è più legato 
alle disuguaglianze e alle contraddizioni del capitalismo globale, raccontandone gli effetti prodotti sul piano dell'immaginario. L'Occidente che entra nelle opere di queste scrittici non è una categoria strettamente geografica, e coincide solo grossomodo con un territorio perimetrabile sulla carta, che ricomprende all'incirca l'Europa dell'Ovest e gli Usa. Più che un'entità geografica, l'Occidente è per loro un'invenzione ideologica ed economica, una costruzione dell'immaginario. Al cuore di questo mito c'è una visione capitalistica del mondo, diversa e per certi tratti opposta rispetto a quella che i personaggi femminili esperiscono vivendo in Albania. In Rosso come una sposa di Ibrahimi la narratrice dichiara esplicitamente che l'Albania si colloca in una posizione di radicale estraneità rispetto all'Occidente: 'Noi, fortunati, ci trovavamo tra due mondi: l'Occidente e l'Oriente. Ma per libera scelta avevamo preferito l'Oriente, tagliando i ponti con l'Occidente" 14 .

Analizzando le narrazioni ambientate nei Balcani, Vesna Goldsworthy e Maria Todorova si sono servite della nozione di "orientalismo"15: da una parte la letteratura occidentale ha fatto dei Balcani un oggetto dell'immaginario, stereotipando la rappresentazione di un'Europa Orientale arretrata, selvaggia e violenta; dall'altra questo stereotipo agisce sulla stessa società balcanica, che a sua volta, di riflesso, ha introiettato l'immagine della propria marginalità nonché la percezione di essere sempre in ritardo rispetto all'economia dell'Occidente. Sia in Rosso come una sposa sia in Vergine giurata è inserito uno stesso episodio narrativo che assume i contorni di un apologo perturbante: gli stranieri fanno incursione tra le montagne dell'Albania a caccia di racconti folkloristici e di testimonianze che abbiano un sapore di esotismo. Nel romanzo di Ibrahimi un giornalista francese intervista la centenaria Meliha per "approfondire la storia del lamento funebre" intonato dalle donne albanesi durante i funerali, e accompagna il racconto della vecchia con esclamazioni entusiastiche ("Bellissimo,

solo alla circolazione dei beni materiali ma a quella delle immagini e alla produzione di tutto il sistema di valori, che determina i rapporti sociali e lo sviluppo delle istituzioni (Polanyi Karl, La Grande Trasformazione [1957], Torino, Einaudi, 2000; ID., Per un nuovo Occidente. Scritti 1919-1958, Milano, Il Saggiatore, 2013).

14. Ibrahimi Anilda, Rosso come una sposa, Torino, Einaudi, 2008, p. 138.

15. Cfr. Goldsworthy Vesna, Inventing Ruritania. The Imperialism of the Imagination, New Haven and London, Yale University Press, 1998; Todorova Maria, Immaginando $i$ Balcani [1997], Lecce, Argo, 2002. Naturalmente il riferimento è alla nozione di “orientalismo" elaborata da Said (SAID Edward, Orientalismo. L'immagine europea dell'Oriente [1978], Milano, Feltrinelli, 2002). 
straordinario, eccezionale', continua a ripetere il francese"16). In Vergine giurata una troupe inglese visita Rrnajë per girare un documentario sul Kanun:

Gli occidentali vengono a saccheggiare le storie di questo Nord definito "primitivo e misterioso". Chi fra i montanari lo vorrà racconterà la propria vita e per questo verrà pagato in soldi stranieri, forse in dollari, forse in moneta tedesca. Ci sono persone che snocciolano storie da vendere, poco importa se vere o no, e gli stranieri comprano le storie che hanno voglia di ascoltare, poco importa se vere o no $^{17}$.

Da queste riflessioni della narratrice Hana emerge il senso di una sfasatura tra la realtà e l'esibizione menzognera di un'identità collettiva, che viene convertita dai visitatori occidentali in un bene di consumo. La differenza culturale diventa esotica. Diventa merce. Le storie di vita hanno un prezzo, e il valore di mercato cresce se il racconto risulta "primitivo e misterioso", sollecitando nel pubblico occidentale la fascinazione per una società fuori dalla storia, in ritardo rispetto al mito di un progresso in continua accelerazione.

Proprio "il male incurabile del ritardo"18, e per di più di un ritardo potenzialmente incolmabile, a dire di Latouche è la miccia che accende il processo di imitazione messo in atto dai Paesi colonizzati, com'è anche l'Albania ${ }^{19}$, nei confronti delle economie capitalistiche dei colonizzatori. Al tempo stesso, però, questo mimetismo caricaturale del modello di sviluppo capitalista, solo apparentemente universale e alla portata di tutti, è uno degli esiti più fallimentari di un'occidentalizzazione che si risolve perlopiù, tragicamente, in una "deculturazione", trasformando ogni valore in merce.

Leggere le narrazioni migranti però permette al lettore italiano anche di capovolgere questa prospettiva: nei testi di Dones e Ibrahimi infatti è l'Occidente a presentarsi, prima di tutto, come un altrove simbolico, come una rappresentazione deterritorializzata dell'homo oeconomicus, che ruota intorno al mito della ricchezza come dispositivo di felicità e canalizza un flusso ininterrotto di desideri, immagini, rappresentazioni e valori alternativi a quelli vigenti nella cultura d'origine

16. Ibrahimi Anilda, Rosso come una sposa, cit., p. 138.

17. Dones Elvira, Vergine giurata [2007], Milano, Feltrinelli, 2015, p. 143.

18. Latouche Serge, L'invenzione dell'economia [2005], tr. it. di Fabrizio Grillenzoni, Torino, Bollati Boringhieri, 2010, p. 84.

19. Per analizzare le ripercussioni letterarie del dominio coloniale italiano sull'Albania, cfr. Bond Emma, Comberiati Daniele, "Narrare il colonialismo e il postcolonialismo italiani. La 'questione' albanese”, in EAD., ID. (a cura di), Il confine liquido. Rapporti letterari e interculturali fra Italia e Albania, Nardò, Besa, 2013, pp. 7-27. 
delle narratrici. Questo ribaltamento produce un effetto "specchio"20: l'immagine dell'economia italiana, e occidentale, viene restituita al rovescio e si traduce narrativamente nell'ottica dello smascheramento. S'invertono i rapporti di potere tra centro e periferia. Il lettore, aderendo allo sguardo inconsueto delle protagoniste di queste storie, considera dall'esterno convinzioni e modelli di comportamento a lui consueti, che però, visti da fuori, sembrano strani e paradossali. Perché, agli occhi delle scrittrici della diaspora albanese, "diversi" sono gli Occidentali. Le loro narrazioni da un lato sollecitano l'identificazione con una prospettiva "altra", che è quella femminile dei codici di comportamento rurali e proto-industriali dell'Albania, dall'altro producono uno straniamento, perché il lettore non guarda solamente, ma è guardato: è il suo mondo a essere indirettamente giudicato, esaminato, preso a temine di confronto. Il lettore fa dunque esperienza di una dislocazione e di un'erranza speculari e opposte a quelle messe in atto nella scrittura. Le storie di Dones e Ibrahimi abitano una lingua "altra", l'italiano, in cui raccontano di donne e "uomini tradotti", per dirla con Rushdie ${ }^{21}$; al contempo, però, la lingua di appartenenza del lettore viene manomessa e smarginata, si allarga a ospitare parole albanesi di cui non esiste il corrispettivo italiano $^{22}$, risuona delle movenze del racconto orale, con cadenze e accenti trasmigrati da una tradizione che non è la propria, ma è radicata nella cultura popolare albanese. Diventa l'irrequieto medium di accesso e di liberazione di una storia che gli appartiene soltanto in parte.

\section{Il tempo NON È DENARO. VERGINE gIURATA Di Elvira Dones}

Il dispositivo narrativo del doppio esprime il senso di una scissione: ai valori della società albanese arcaico-rurale, basata sulla micro-cellula della famiglia, cadenzata dai ritmi immutabili della natura e della tradizione contadina, si oppongono i controvalori economici di un Occidente espanso in movimento perpetuo. Così il romanzo Vergine giurata è segnato dall'ambivalenza, a partire

20. Mengozzi Chiara, Narrazioni contese. Vent'anni di scritture italiane della migrazione, Roma, Carocci, 2013, p. 8.

21. Rushdie Salman, Patrie immaginarie, tr. it. di Carola di Carlo, Milano, Garzanti, 1991, p. 22.

22. Parole come kurva ("prostituta", utilizzata per definire la donna che ha commesso adulterio o che ha avuto rapporti sessuali prima del matrimonio, macchiando indelebilmente l'onore della sua famiglia) o raki (la grappa albanese che accompagna i brindisi rituali) ricorrono nelle opere italiane delle scrittrici albanesi e veicolano una carica identitaria che si lega a una precisa antropologia: sono termini refrattari alla traduzione, che non possono essere "detti" in un'altra lingua. 
dalla scelta della focalizzazione fissa sulla protagonista Hana, che è una burrnesh - una "vergine giurata". Per sottrarsi al destino di moglie-schiava imposto alle donne nelle montagne dell'Albania, seguendo la legge arcaica del Kanun Hana ha assunto un'identità maschile, ha acquisito il nome di Mark e ha scelto di condurre la vita con l'autonomia concessa a un uomo, rinnegando però radicalmente la propria femminilità. Sospesa tra maschile e femminile, Hana è al confine tra generi e mondi differenti. La narrazione comincia nel momento in cui, vestita da uomo, Hana approda nell'aeroporto internazionale di Washington. Da qui inizia il suo viaggio nel "mondo lontano" che va al "ritmo della vita moderna" 23 . Come nel più tradizionale dei Bildungroman, la sua ricerca è siglata da un bappy end, suggellato dall'inserimento nel produttivo mercato del lavoro statunitense. La riconquista di un'identità femminile in progress passa attraverso un processo di graduale omologazione; nel contesto del mercato globalizzato, le scelte di consumo diventano per Hana altrettanti veicoli di emancipazione. Eppure, mentre si immerge nel paesaggio artificiale della metropoli, punteggiato di centri commerciali e di ristoranti vietnamiti, Hana sperimenta lo choc di abitare un tempo economico e cumulativo. Un tempo distorto, che entra in conflitto con il tempo naturale di "lassù": nel suo minuscolo paese tra le montagne dell'Albania, "il tempo e il luogo vivevano insieme"24; era netta la distinzione tra prezzo e valore; il denaro non era immateriale, ma aveva la concretezza preziosa delle monete che le donne vestite a festa portavano "appese in fronte" e "facevano tintinnare in una gioiosa cacofonia" 25 . Per l'uomo occidentale invece, si sa, il tempo è denaro, e la cugina Lila, con "la frenesia negli occhi" 26 , lo ricorda ossessivamente ad Hana ripetendo che "il tempo non va sprecato" 27 , perché "in America si deve vivere nel presente" Hana non ha fretta. "Non c'è fretta" è il refrain che scandisce gli snodi più significativi del romanzo in un'insistita tessitura di ripetizioni e di riprese variate $^{29}$. La frana della continuità incide sulle forme della narrazione: il tempo diventa reversibile e la linearità del racconto si spezza. La storia dell'integrazione di Hana negli Stati Uniti si esaurisce in un giro di mesi circostanziato e misurato con precisione (dall'ottobre 2001 alla primavera-estate del 2003), ma la sintassi

23. Dones Elvira, Vergine giurata, cit., p. 13.

24. Ibid., p. 43.

25. Ibid., p. 171.

26. Ibid., p. 49.

27. Ibid., p. 44.

28. Ibid., p. 52.

29. Cfr. Ibid., pp. 41, 43, 45, 47, 164, 182, 196. 
paratattica è sempre lacerata dalle analessi, assediata da vertiginosi ritorni del rimosso, dai riaffioramenti di un passato che incalza il presente e lo slabbra.

\section{Locale Versus globale. Rosso come una sposa di Anilda Ibrahimi}

In Rosso come una sposa di Ibrahimi il narratore ha un doppio statuto. Nella prima metà del romanzo è esterno e onnisciente, mentre il fuoco della narrazione si concentra sul personaggio di Saba, che la madre Meliha dà in sposa al vedovo della sua prima figlia Sultana. Nella seconda parte il narratore si individualizza ed entra nella vicenda che racconta: la sua voce coincide con quella di Dora, nipote di Saba, che in prima persona prosegue il racconto retrospettivo della propria storia e di quella delle donne della sua famiglia. Dora e Saba, dunque, sono protagoniste di una narrazione che attraversa quattro generazioni di donne e si svolge principalmente su due luoghi: Kaltra, un piccolo villaggio fra le montagne, e Valona, la città. L'incipit del romanzo trasporta allora il lettore in un tempo senza tempo: “Arriva in una mattina di settembre, in un'arsa stagione dove le piogge tardano a venire. È vestita tutta di rosso. Come il sangue. Come un sacrificio umano dato in dono agli dèi per propiziare la pioggia. Come una

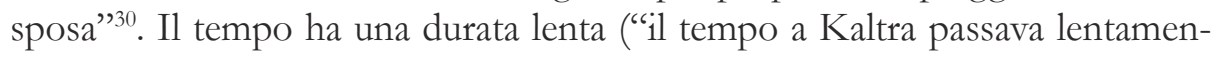
te" ${ }^{\prime 1}$, si legge nel diciottesimo capitolo): l'uso di un presente storico e pluriprospettico $^{32}$, che caratterizza tutta la Parte prima del romanzo, cumula su di sé lontananza e prossimità, esprime il senso mitico di un tempo ciclico e premoderno, scandito dal susseguirsi delle stagioni, dalle piogge, da antichi e crudeli riti sociali. Lo spazio è quello chiuso e immobile del paese di Kaltra, che vive di agricoltura. Si basa cioè su una economia contadina e agricola, saldamente legata alla terra e ai ritmi della natura, in cui la produzione non è finalizzata al profitto ma alla sussistenza della famiglia. La famiglia non rappresenta solo una comunità sociale coesa, ma anche un'unità fondamentale di organizzazione del lavoro e dei guadagni, la cui amministrazione, all'interno dell'oikos, è prerogativa esclusiva delle donne. Generazione dopo generazione per i contadini di Kaltra "il mondo inizia e finisce qui" 33 , tra le montagne che "si alzavano come coltelli ben affilati", quasi a voler "tagliare fuori dal mondo

30. Ibrahimi Anilda, Rosso come una sposa, cit., p. 5.

31. Ibid., p. 94.

32. Cfr. Sorella Antonio, "Per un consuntivo degli studi recenti sul presente storico", in Studi di grammatica italiana, n. 12, 1983, pp. 307-319.

33. Iвrahimi Anilda, Rosso come una sposa, cit., p. 106. 
queste esistenze" ${ }^{34}$. Questo microcosmo dilatato "sembrava non essere in contatto con niente e nessuno, tranne che con il tempo" ${ }^{35}$ : la precisione nella rappresentazione dello spazio sociale del paese e l'esattezza della localizzazione geografica si combinano con un'estrema indeterminazione, che sembra obbedire a un'esigenza di mitizzazione e di favola. Ma è una favola di violenza e sopraffazione: il mondo di Kaltra è spietato e, a sua volta, diventa oggetto di un altrettanto spietato processo di spersonalizzazione e di colonizzazione. La modernità, con il suo tempo lineare e con il suo immaginario economico, muta i cromosomi del paese fino a condurlo alla dispersione, tramutando una "civiltà della permanenza" in una "civiltà del moto perpetuo"36 e della diaspora. La Parte seconda si apre sulla scena di un nuovo matrimonio, celebrato nel 1971:

Il giorno del suo matrimonio mia madre indossava un tailleur beige dal taglio semplice e nei capelli non portava nessun velo. [... ] Una volta arrivato davanti a casa di nonna Saba, la madre di mio padre, il corteo si fermò di colpo ${ }^{37}$.

II tempo verbale al presente della Parte prima viene soppiantato ora dall'imperfetto della rievocazione e dal passato remoto, il tempo storico dell'azione puntuale. Questa opposizione dei tempi verbali acquista un rilievo antropologico: al tempo mitico e immobile dei primi ventitré capitoli, ambientati a Kaltra, si sostituisce un tempo progressivo e economico. L'azione si trasferisce infatti nella città di Valona e poi a Tirana, dove si spezza la solidarietà tra uomo e natura. Lo spazio si allarga e, insieme, diventa periferico. Chi si sposta in città fa esperienza di una sorta di vertigine ottica: lo spazio metropolitano si rimpicciolisce di colpo; Tirana e Valona si riducono a province di un Occidente globalizzato. Sullo sfondo della narrazione si proietta un luogo terzo e ulteriore, lo spazio dell'enunciazione, alluso ma non rappresentato: è Roma, la "location of culture" 38 , l'altrove da cui la narratrice Dora parla e nel quale è capitata accidentalmente. "Presi la mappa del mondo, chiusi gli occhi e puntai il mio dito cieco su Roma!" ${ }^{39}$ : Roma è solo per avventura Roma. Si disegna sulla

\section{Ibid., p. 17.}

35. Ibid.

36. L'antitesi antropologica tra una "civiltà della permanenza" e una "civiltà del moto perpetuo" è al centro del Montezuma di Calvino. Cfr. Calvino Italo, Prima che tu dica "pronto" [1984], Milano, Mondadori, 2011, p. 184.

37. IвRAhimi Anilda, Rosso come una sposa, cit., pp. 123-124.

38. L'espressione è ripresa dal titolo del saggio di Homi Bhabha del 1994, The Location of Culture. Cfr. Bнавна Homi, I luogbi della cultura, cit.

39. IвrAhimi Anilda, Rosso come una sposa, cit., p. 257. 
mappa dell'Occidente come il toponimo di una geografia fantastica e livellata, e il suo nome riassume simbolicamente i nomi di tutte le "capitali luccicanti" e di tutte le merci che popolano l'immaginario di Dora per accumulo e senza differenze di valore. Roma è un "oltre" come l'America, è un orizzonte del desiderio, come lo sono le marche dei "vestiti arrivati dall'Occidente: ultima moda" ${ }^{41}$ o delle "macchine di lusso rubate in Occidente, [...] Mercedes, Porsche, Range Rover" ${ }^{42}$, e i nomi dei quotidiani internazionali che per il pazzo di Valona sono i nomi stessi delle città:

Mi veniva in mente lo scemo del mio quartiere a Valona, che nei pomeriggi torridi d'estate, seduto sui muretti, strillava: "Vado a Washington Post, a New York Times, me ne vado, razza di merda, me ne vado da qui”. E se n'è andato per davvero. A New York, senza Times, s'è accontentato. Come si accontentano quelli che se ne vanno ${ }^{43}$.

Il romanzo di Ibrahimi ha una struttura ciclica: si chiude con la narrazione che la nipote Dora scrive come una sorta di "lamento funebre" per la nonna Saba, nella convinzione che soltanto l'atto della scrittura possa riannodare i legami spezzati e dare significato e "valore" alla vita passata. In Rosso come una sposa, in Vergine giurata, e più in generale nelle opere delle scrittrici migranti, i personaggi delle donne straniere, come ha scritto Silvia Contarini, svolgono "ruoli positivi per la comunità e comunque di rilievo, perché sono le donne a tramandare le storie o a ricucire gli strappi" ${ }^{44}$. Impegnandosi a narrare la storia di tutta una collettività, si fanno portatrici di una tendenza costitutiva dell'umano che fa resistenza alla logica della performance e all'ottimizzazione del profitto: la tendenza al dono e alla trasmissione della memoria familiare.

Claudia CARMina Università di Palermo

40. Ibid.

41. Ibid., p. 241.

42. Ibid., p. 255.

43. Ibid., p. 257.

44. Contarini Silvia, "Narrazioni, migrazioni e genere", in QuAQuARELLI Lucia (a cura di), Certi confini. Sulla letteratura italiana dell'immigrazione, Milano, Morellini Editore, 2010, p. 155. 